\title{
Human Factor Case - Tool for Systematic Identification and Management of Human Factor Issues for Air Traffic Management Project
}

\author{
R. Štecha*, J. Šulc, V. Voštová \\ Department of Transportation Sciences, Czech University of Technology, Prague, Czech Republic \\ *Corresponding author: stechric@fd.cvut.cz
}

DOI: $10.2478 / \mathrm{v} 10158-012-0013-4$

ABSTRACT: This paper presents human factor as a crucial element of flight safety. It is a generally known fact that human factors play an important role in incidents and accidents in aviation, especially regarding pilots and technical personnel, as well as air traffic controllers. The amount of air transport grows dramatically year by year as the saturation of air traffic service airspace similarly increases, filled with sporting (amateur) aviation, military, and other flights (aero-medical, sightseeing flights, etc.). The relationship between human performance and safety has been a long-standing issue with Air Traffic Control authorities, since human performance is considered to be a critical determinative for Air Traffic Management safety.

KEY WORDS: $\quad$ Human Factor, Human Performance, Air Traffic Management.

\section{INTRODUCTION}

At sharp end of performance in Air Traffic Management (ATM), professionals manage their own performance at a tactical level - controllers, supervisors, engineers, etc. Behind the scenes, other groups of professionals and means contribute to the improvement of human performance at a more strategic level. These elements use various principles and methods for measuring and influencing human performance - directly or indirectly. Three “enablers” of human performance in ATM are noteworthy (see Figure 1).

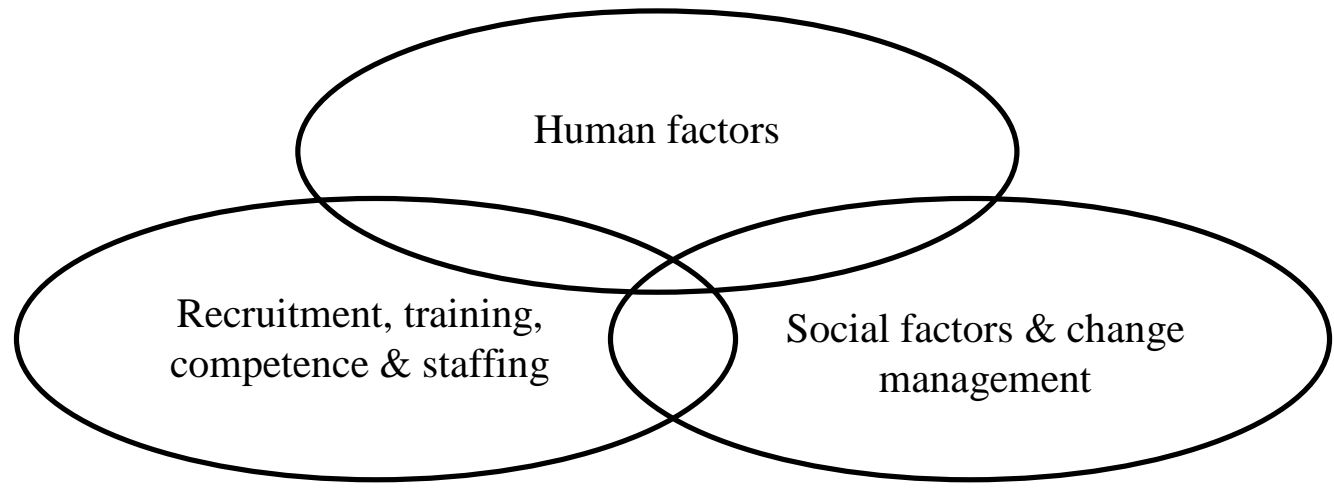

Figure 1: Delivering human performance benefits. 
- Human Factors (HF) is a design-oriented discipline and profession which develops and applies the knowledge about the performance of people at work towards the design of the work. It focuses on the task requirements, the equipment and technology people use, the rules and procedures they work under, the ways in which they communicate, and the physical and organisational environment in which they operate. HF focuses mostly on "fitting the job to the person". HF issues are also classified as HF Impacts on Human Performance (see Figure 2).

\begin{tabular}{|l|l|l|}
\hline \multicolumn{1}{|c|}{ HUMAN FACTOR } & \multicolumn{1}{|c|}{ IMPACTS } \\
\hline $\begin{array}{l}\text { The HF Case is a process to } \\
\text { systematically identify and } \\
\text { treat HF issues and benefits } \\
\text { during an ATM project } \\
\text { throughout its lifecycle, } \\
\text { from concept to } \\
\text { decommissioning. }\end{array}$ & $\begin{array}{l}\text { Human Performance in } \\
\text { ATM focuses on all job } \\
\text { related aspects at the } \\
\text { individual, group and } \\
\text { organisational levels that } \\
\text { can impact upon human } \\
\text { capability to successfully } \\
\text { accomplish a wide variety } \\
\text { of tasks and job } \\
\text { requirements including the } \\
\text { management of related } \\
\text { changes. }\end{array}$ \\
\hline
\end{tabular}

Figure 2: Relation between Human Factors issues and Human Performance.

- Recruitment, training, competence, and staffing are the primary concerns of human resource management (HRM) and occupational and organisational psychology. The priorities are to attract and retain talented and competent staff, as they will ultimately determine the success and sustainability of the organisation. HRM and psychology focus more on "fitting the person to the job".

- Social factors and change management refers to a social dialogue and change process, which will pave the way forward for future concepts if accepted and recognised by all parties involved and affected by the changes.

All three enablers secure the compatibility or "fitness" between people, their work, and the organisation, regardless of the differing focus of each issue. They overlap in the introduction of large-scale changes, such as the Single European Skies ATM Research (SESAR) in Europe, NextGen in the US, and Automatic Dependent Surveillance-Broadcast (ADS-B) in Australia and Canada.

\section{HUMAN FACTOR CASE}

The HF Case is a management tool which systematically identifies and manages HF issues and manages HF issues for an ATM project. It can be divided into five stages (see Figure 3). 


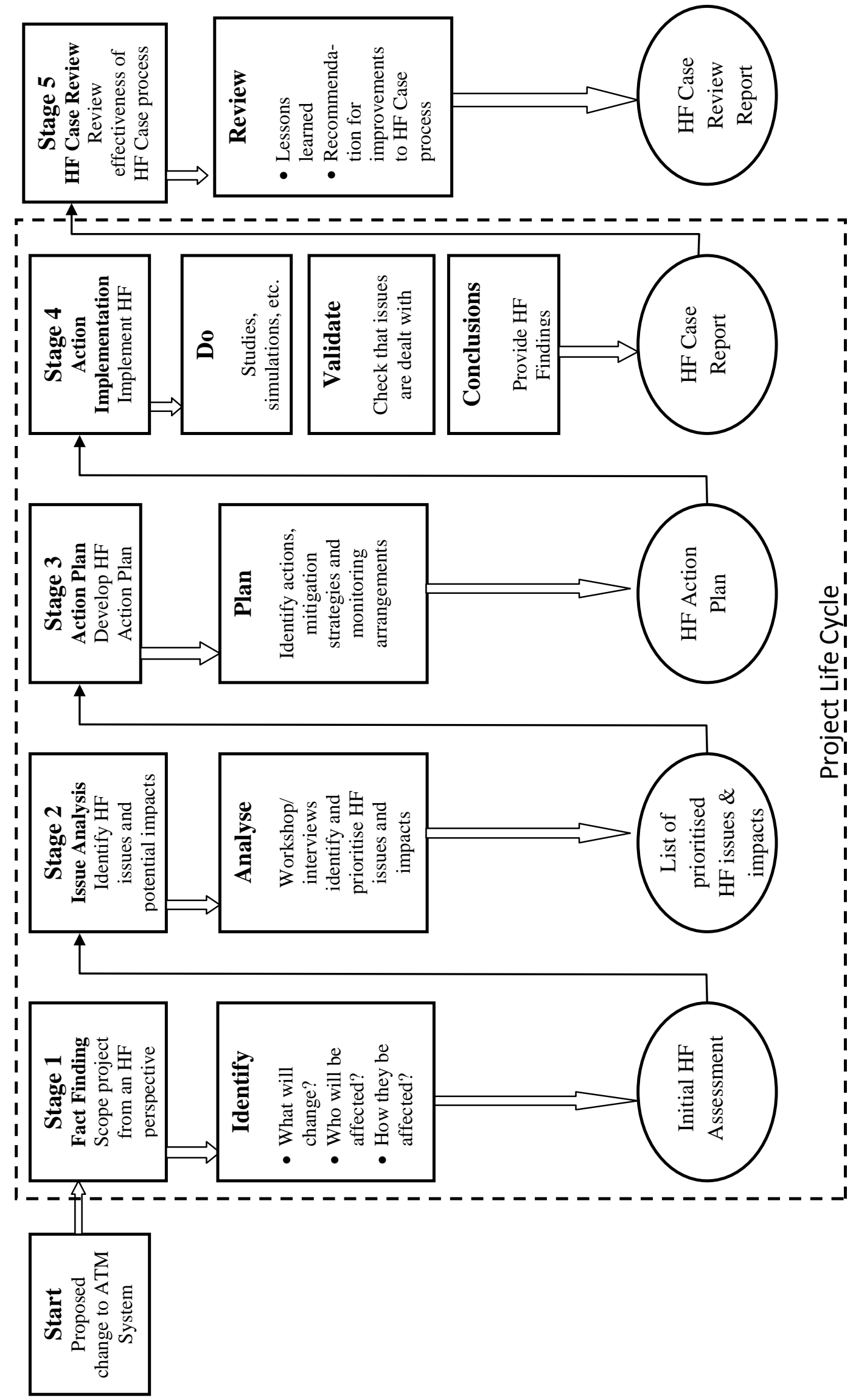

Figure 3: Stages of Human Factors Case. 
It provides and enables:

- A framework to address HF issues

- The application and integration of subject matter expertise and HF knowledge

- A comprehensive qualitative analysis

The HF Case process provides:

- An explicit way to manage HF issues

- A checklist and traceability for HF issues as the project evolves

- Ownership within the project team for HF

- Facilitates decision making to justify resources and budget for HF

- Minimises the risk of HF issues popping up at a critical stage

This means that HF Case is aimed at:

- Programme and Project Managers - Providing assurance that HF is integrated into the project and there is awareness among the project team.

- Validation - Allows them to track HF issues from simulations and experiments.

- Safety - The HF Case is complementary to a Safety Case and may help them identify safety relevant issues for the Safety Case.

- Training and Staffing - Develops an awareness of staffing and training issues that may need to be tackled later in the project life cycle.

\subsection{Human Factor Pie}

To facilitate the identification of issues related to human factors within a project, HF issues are classified into the following six main categories, called the "HF Pie". The HF Pie underlies the general approach to the identification, assessment and monitoring of HF issues relevant to the project. They are displayed in the diagram below (Figure 4). By investigating each element of a given category, the analytic team can identify issues relevant to a specific project.

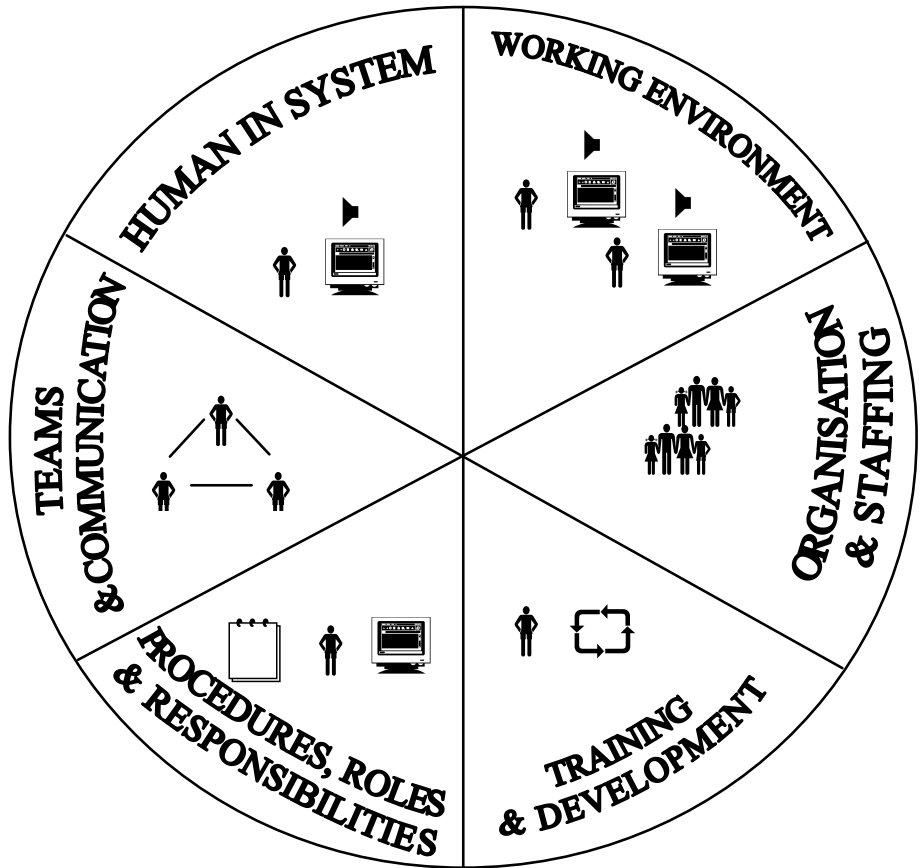

Figure 4: Human Factors Pie. 


\section{HUMAN PERFORMANCE CHALLENGES FOR FUTURE AIR TRAFFIC MANAGEMENT}

The future of Air Traffic Management (ATM) will depend on the ability of the industry to handle a number of critical challenges concerning human performance. Six key challenges are outlined below.

\subsection{Designing proper technology}

Future technology will be a step change from current technology. The focus will shift to collaboration across sectors and centres, and between ground and air to support the shared "situation awareness". Tools will also need to accommodate more advanced planning and look-ahead time, while supporting the flexibility required to deal with unplanned situations. At the same time, it must be ensured that it is possible to handle unexpected disturbances and degraded modes safely. Crucially, the automation must keep the human operator in the loop to be able to maintain control - and thereby safety - in all circumstances.

\subsection{Selecting the right people}

Major technological and organisational changes may require changes to the type and number of people required to operate the business effectively. This may require changes to manpower planning, recruitment, and selection to ensure that we have the right people, at the right numbers, at a time.

\subsection{Organising the people into the right roles and responsibilities}

A new collaborative approach to ATM will result in new roles and responsibilities for controllers and engineers, as well as for other ground staff. In light of increased delegation, such changes will extend to flight crews. Roles are likely to be more fluid than is the case today. The human performance implications of transitioning between roles must be clearly understood and managed.

\subsection{Ensuring that the people have the right procedures and training}

New technology, people, roles, and responsibilities all impact the training and procedures required, both for new and existing staff. Competencies will also need to be maintained for old skills that may be used more rarely in light of new technology, but are still critical when needed. The new collaborative approach to ATM may require new collaborative approaches to training.

\subsection{Managing human factors processes at a project and ANSP level}

Consideration of human performance issues requires human factors to be fully integrated with system development and safety management. The management goals are to meet the demands for efficiency, enabling capacity gains and safety improvement. Performance indicators can be useful here, to benchmark and quantify the maturity of human performance assurance at an organisational level. 


\subsection{Managing the change and transition process}

A successful project depends on a successful change and transition process, where the social, cultural, and demographic factors impacting performance are considered alongside the technical and procedural factors.

ATM today is one of very few "high reliability industries". Throughout the major changes of the future, we need to keep it this way. Strategic, management-level approaches are necessary to maintain performance throughout every stage of the design, development and implementation process, before reaping the performance and safety benefits during the operations phase. The right management systems and organisational culture, including safety culture, will help to ensure that the capacity, efficiency and safety benefits expected are realised.

\section{HUMAN PERFORMANCE AND ORGANISATIONAL BUSINESS PERFORMANCE}

Compared to the other high-hazard industries, such as chemical processing, nuclear power, and even aviation more generally, air traffic management still remains "human-centred". Despite advances in technology, ATM is still critically dependent on the day-to-day performance of highly skilled front-line personnel, such as controllers, engineers, supervisors, and other operational staff. Operational personnel safely and efficiently handle millions of flights, and effective human performance at the front line ensures this happen. Human performance solutions are required to bring the people, procedures, and equipment together effectively (see Figure 5) in order to make running the business more efficient and safer.

In terms of SAFETY, 2006 and 2009 were the safest years on record worldwide. 2008 was the fifth consecutive year without ATM-related accidents in Europe. Traffic growth is the key challenge to maintaining such a record, because when traffic doubles, risk is squared. The European SESAR programme aims to improve the safety performance by a factor of 10 by 2020. Clearly, the human element will be critical to ensuring that safety is maintained.

The industry needs to gain additional CAPACITY and reduce delays to meet the demands of traffic growth. The SESAR programme aims to enable a 3fold increase in capacity. Again, this can only be achieved when those managing the traffic are considered.

A third priority is EFFICIENCY. SESAR aims to reduce the costs of ATM services to airspace users by $50 \%$.

These improvements make significant demands on human performance, but the financial benefits will be significant. According to the European ATM Master Plan, the savings attributable to direct ATM cost reduction, capacity gain and departure delay savings, as well as predictability improvement in case of low visibility conditions, is around €19bn for commercial airlines by 2020, with an additional $€ 12$.5bn of savings through passenger travel time.

To achieve the right fit, it is necessary to assert proper professional resources in the organisation. Whilst HRM and platforms for social dialogue are more commonplace, HF expertise in ATM is less so. Nevertheless, a number of ANSPs now have specific teams of qualified human factors specialists, integrated into design, selection, training, and safety functions. Some also have human performance teams comprising operational and engineering staff with a special interest in the domain. As human performance issues are a key driver of ATM performance, they need to receive considerable attention in planning, design, operations and maintenance, and should be treated as seriously as other business-critical functions. 


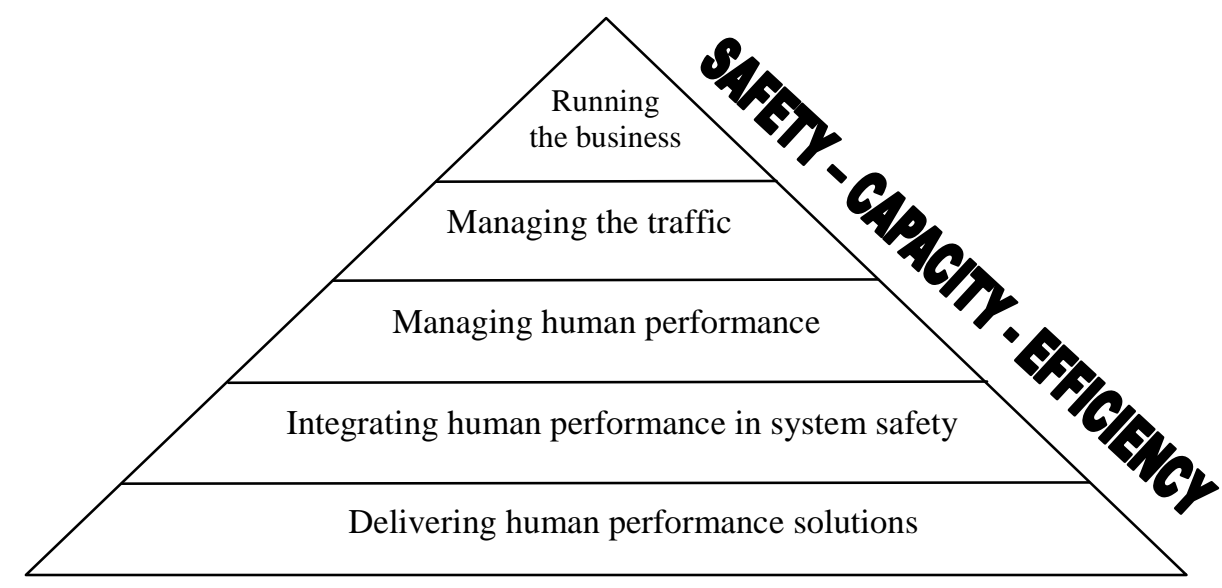

Figure 5: Human performance and organisational business performance.

\section{UNDERSTANDING HUMAN PERFORMANCE}

Human performance at work has been the subject of intense research in several disciplines for decades. Much is now known about how people perform tasks, and why they perform them in the way that they do. But much of this is hidden away in books and journals for academics and specialists.

Human performance, in the context of ATM, refers to the adequate performance of jobs, tasks, and activities by operational personnel - both individually and together. As a domain, human performance focuses on optimising the people element in complex work systems, such as air traffic management. Designing for human performance and managing human performance involves the application of knowledge gained from research and practice in human factors, psychology, and management.

Human performance depends on both the person and the context of work. Capability refers to the basic characteristics of the individual, e.g., aptitude, abilities, skills, physical capabilities, knowledge, experience, and health. Capabilities are assessed during selection and promotion, shaped and enhanced via training, and considered in the design of jobs, task/activities, systems, and tools.

Motivation and attitude influence the use of the person's capabilities. While a person's motivation varies, it is critical in ensuring that capabilities are fully realised in human performance. Motivation, attitude and trust can be improved significantly with the right approach.

The systems, organisation, and environment provide the opportunity for good performance, given sufficient capability and motivation, and include systems and technology, the design of the job and tasks, the workplace environment, training and procedures, and management and support. These can be designed and managed directly.

All three components have to be considered carefully. Even very highly capable individuals will not perform well if motivation is low or if the system, organisation or environment (e.g., training and procedures) are poor. Similarly, even the most motivated person, with good training and procedures, may not perform well if capabilities are poorly matched to the job requirements. Human performance can vary, positively or negatively, depending on the capability, motivation, system support, organisation, and environment. 


\section{CONCLUSION}

In ATM, human performance and safety are inextricably linked. While the disciplines involved in improving human performance are themselves continually developing and improving, the techniques to identify and resolve these issues exist. They need to be embraced and integrated into the systems developers' and project managers' "mindsets" and practices.

As ATM continues to evolve in terms on NextGen improvements in the US, and Single Sky, Functional Airspace Blocks and SESAR in Europe, this will create new challenges for human performance and safety, as well as generating system performance advantages.

It is perhaps obvious that safety depends on human performance, and that they need to work together. What is less clear sometimes is how they can work together in practice. This paper has aimed to describe that there are techniques, approaches, and data sources which allow a strong synergy to take place between these two disciplines which share a common goal. It is hoped that it may encourage ANSPs, their managers, engineers, safety, and human factors professionals and researchers, to find effective ways to work together so that ATM can continue to enable aviation to remain the safest system of public transport, now and in the future.

\section{REFERENCES}

Kirwan, B. \& Devine, J., 2010. Human Performance in Air Traffic Management Safety A White Paper, pp. 4 - 21.

Isaac, A. et al., 2002. Short Report on Human Performance Models and Taxonomies of Human Error in ATM (HERA), pp. 3 - 10.

Eurocontrol [online]. Eurocontrol - European Organisation for the Safety of Air Navigation, (C) 2001 - 2011. Retrived from: http://www.eurocontrol.int/humanfactors/public/ site_preferences/display_library_list_public.html

SKYbrary [online]. Retrieved from: http://www.skybrary.aero/index.php/Portal:Human_ Performance

Eurocontrol Training Zone [online]. Retrieved from: https://trainingzone.eurocontrol.int 